\title{
Salmonella enterica serovar Typhimurium strain lacking pmrG-HM-D provides excellent protection against salmonellosis in murine typhoid model
}

\author{
Vidya Devi Negi, Santanu Singhamahapatra, Dipshikha Chakravortty* \\ Centre for Infectious Disease Research and Biosafety Laboratories, Department of Microbiology and Cell Biology, \\ Indian Institute of Science, Bangalore 560012, Indiaailable online 4 June 2007
}

\begin{abstract}
The superiority of live attenuated vaccines in systemic salmonellosis has been proven over killed and subunit vaccines, because of its ability to induce protective cell mediated immunity by CD8+ T cells. A live attenuated Salmonella enterica serovar Typhimurium vaccine has been developed by systematic site directed deletion of the pmrG-HM-D chromosomal genomic loci. This gene confers involved in antimicrobial peptide resistance and is involved in LPS modification, both of which are the major immune evasive mechanisms in Salmonella. The efficacy of the newly developed strain in inducing protection against mortality after challenge with the virulent wild type Salmonella typhimurium 12023 was evaluated in mice model of typhoid fever. Animals were immunized and then boosted on days 7 and 14. Following challenge with virulent S. typhimurium 12023, organ burden and mortality of vaccinated mice were less compared to non-immunized controls. The vaccine strain also induced elevated CD8+ T cells in the vaccinated mice. This multiple mutant vaccine candidate appears to be safe for use in pregnant mice and provides a model for the development of live vaccine candidates against naturally occurring salmonellosis and typhoid fever.
\end{abstract}

Keywords: pmrG-HM-D mutant; Salmonella tyhpimurium; Vaccine

\section{Introduction}

Salmonella typhi, a causative agent of typhoid fever is raising a major threat due to its potential use in bioterrorism [1] and the non-availability of an efficient candidate vaccine. About 21.6 million people have suffered from typhoid and over 216,500 have succumbed to it, globally, in the year 2000 alone [2]. The incidence of typhoid is high $(>100$ cases per 100,000 population each year) in south-central Asia, southeast Asia and possibly southern Africa (10-100 cases per 100,000) [2]. The increased appearance of antibiotic resistant strains of Salmonella further complicates the situation [3]. The Salmonella vaccine strains created so far had mutations in the metabolic genes or pathogenicity islands. Salmonella harboring mutations in SPI2 [4], aro A [5], hrtA [6] have been tested as a vaccine candidates in both animal models and humans. These vaccine candidates have not been able to fully protect the animals. The only licensed attenuated live oral typhoid vaccine, S. typhi strain Ty21a, is well tolerated and immunogenic, but requires three or four spaced doses of 2-6 $\times 10^{9}$ CFU given every other day, an important practical shortcoming [7]. Ty800, a vaccine strain, where phoP gene was knocked out, exhibited excellent protection in human volunteers [8]. Creation of vaccine strain which will provide full protection against typhoid fever requires greater understanding of immune evasion strategies used by Salmonella. The intricate lifestyle of Salmonella inside the host cells, makes this pathogen a better survivor. By evading the immune mechanism, Salmonella survives in the host and can cause life-threatening infec- 
tions. Though studies have been done to look at the immune profile of T cells [9], cytokine level [10] and the antibody response [11] in murine model of Salmonella, none of these could not rescue the mice from Salmonella infection, suggesting that the immunity is not protective. Till date, the candidate antigen of Salmonella which can evoke a long lasting protective immunity remains unidentified. As vaccine is the only answer to combat this disease, thought should be given to rationally develop a vaccine with longterm protective immunological memory against Salmonella infection.

The key to success for many bacteria in causing infection is colonization of host tissues. An enteric bacterium, such as Salmonella, gains entry through the oral route and survives the harsh environment of the intestine. At the intestinal mucosa, these bacteria encounter host defense mechanisms including antimicrobial peptides (AMPs), which are cationic, amphipathic molecules that kill bacteria by membrane permeabilization. Within the intestine, AMPs are secreted into the lumen by Paneth cells located in the base of intestinal crypts. AMPs are also found within phagocytic cells located in the intestinal submucosa. The ability of Salmonella to survive within the host intestine and within professional phagocytes is likely to depend, at least in part, on mechanisms of resistance to AMPs. The Pmr systems in Salmonella which includes pmrHIFJKLM, pmrD, pmrA-B, pmrE and $p m r G$ are known to modify LPS, confer resistance to antimicrobial peptides and to regulate other two component regulatory system [12]. Because of the intricate network among pmr systems, we have created a multiple mutant where the Salmonella is devoid of $p m r D, p m r G$ and the pmrHIFJKLM ( $p m r H-M)$ operon. This multiple mutant Salmonella strain $(\triangle p m r G$ $H M-D)$ is highly attenuated and does not cause disease even at higher doses on oral and intraperitoneal administration. A single dose of this vaccine candidate could protect the mice very efficiently in the challenge experiments, suggesting its ability to evoke a strong protective response in the host.

\section{Materials and methods}

\subsection{Bacterial strains, media and growth conditions}

Salmonella enterica serovar Typhimurium strain NCTC 12023 was used as wild type strain (kindly gifted by Prof. M. Hensel). Bacteria were routinely cultured in LB broth and on LB agar plates. If required to maintain plasmids, carbenicillin $(50 \mu \mathrm{g} / \mathrm{ml})$ and/or chloramphenicol $(50 \mu \mathrm{g} / \mathrm{ml})$ were added.

\subsection{Construction of pmrG-HM-D mutant of Salmonella typhimurium}

$\Delta p m r G-H M-D$ was constructed by one-step deletion strategy of Datsenko and Wanner [13]. Salmonella transformants

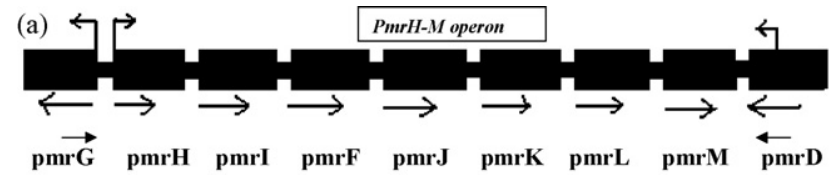

(b)

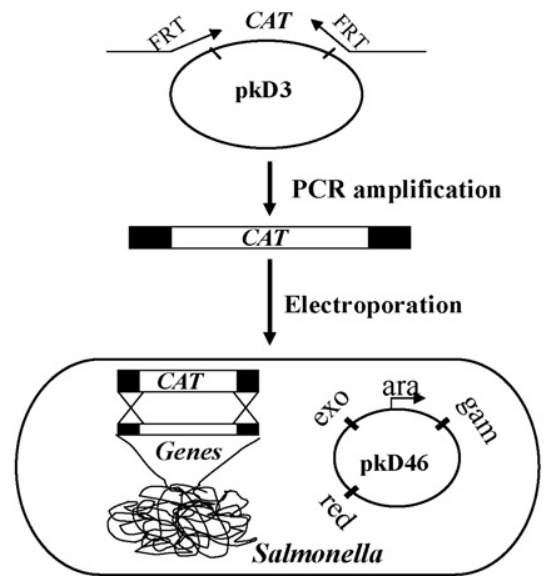

Fig. 1. (a) Genetic make up of pmrGH-MD genes in WT Salmonella. $p m r G$-putative aluminum inducible gene, $p m r H$-putative LPS modification and polymyxin B resistance gene, pmrI-putative sugar transferase, $p m r F$ putative transferase, $p m r J$-putative cytoplasmic, $p m r K$-effect polymyxinB resistance and LPS modification, $p m r L$-putative mallinin resistant gene, pmrM-putative inner membrane protein, $p m r D$-PolymyxinD resistant gene. Thin arrow indicates the region used for designing primers to create the knock out. (b) One step deletion strategy was applied to construct the multiple mutant.

carrying a red helper Plasmid (pKD46) were grown in LB with carbencillin and $10 \mathrm{mM} \mathrm{L}$-arabinose at $30^{\circ} \mathrm{C}$. When the cells reach an OD $(600 \mathrm{~nm})$ of $0.4-0.5$, electrocompetent cells were made by washing three times with ice-cold autoclaved milli Q water and $10 \%$ glycerol. The chloramphenicol cassette (CAT) of pKD3 was amplified with primers having overhangs of flanking regions of pmrGM$H D$ (Fig. 1). This PCR product was electroporated into Salmonella having pKD46. Immediately after electroporation $1 \mathrm{ml}$ of SOC was added and incubated at $37^{\circ} \mathrm{C}$ for $1 \mathrm{~h}$ before plating on to the LB-agar plates containing chloramphenicol $(50 \mu \mathrm{g} / \mathrm{ml})$. Mutants were selected by virtue if their ability to grow on the antibiotic plates and confirmed by colony PCR using specific confirmatory primers (Table 1).

Table 1

Showing primer used for construction and confirmation of mutant

\begin{tabular}{ll}
\hline Deletion & \\
Forward primer & $5^{\prime}$-ATTTTGTATATCGGCACTGAAGGCTTAC- \\
& CCAGCGC-GTGTAGGCTGGAGCTGCTTCG-3' \\
Reverse primer & $5^{\prime}$-AAAATGATCGCCGAGGCGAATTCCATGA- \\
& TATTACT-CATATGAATATCCTCCTTA-3' \\
Confirmation & \\
Forward primer & $5^{\prime}$-GACCTGCCCGCAGAAAAC-3' \\
Reverse primer & $5^{\prime}$-AAGAAATCGCATTATGTC-3' \\
\hline
\end{tabular}




\subsection{Eukaryotic cell lines and growth conditions}

RAW 264.7 cells (gifted by Prof. Anjali Karande, Dept of Biochem., IISc) and INT407 (NCCS, Pune) were grown in Dulbecco's modified Eagle's medium (DMEM) containing $10 \%$ fetal calf serum (FCS, Sigma) and $10 \mathrm{mM}$ sodium pyruvate at $37{ }^{\circ} \mathrm{C}$ and $5 \% \mathrm{CO}_{2}$. Colo205 (NCCS, Pune) cells were grown in RPMI-1640 supplemented with 10\% FCS and $10 \mathrm{mM}$ sodium pyruvate at $37^{\circ} \mathrm{C}$ and $5 \% \mathrm{CO}_{2}$.

\subsection{Isolation of peritoneal macrophage}

Six- to eight-week old female BALB/c mice were intraperitoneally injection of $5 \mathrm{ml}$ Brewer's thioglycolate broth (HiMedia, India). After 4 days, the macrophages were isolated from peritoneal cavity by injecting $5 \mathrm{ml}$ ice-cold RPMI-1640 medium and aspirating after 2 min under sterile conditions.

Peritoneal cells were washed once $\left(300 \mathrm{~g}, 5 \mathrm{~min}, 4{ }^{\circ} \mathrm{C}\right)$ and then cultured $\left(1 \times 10^{6}\right.$ cells $\left./ \mathrm{ml}\right)$ in RPMI medium containing $10 \%$ FCS in 24 -well plates. After $3 \mathrm{~h}$ of incubation, non-adherent cells were removed to enrich peritoneal macrophages.

\subsection{Growth curve of $S$. typhimurium in LB, minimal medium (MM) and F medium (FM)}

Overnight grown cultures of Salmonella wild type (WT) and $\Delta p m r G-H M-D$ strains were subcultured (1\%) into $25 \mathrm{ml}$ of LB, MM and FM and grown at $37^{\circ} \mathrm{C}$ in triplicates with constant shaking at $115 \mathrm{rpm}$. The optical density of the cultures at $600 \mathrm{~nm}$ was measured using spectrophotometer every hour for $24 \mathrm{~h}$. The growth curves were plotted as optical density against time.

\subsection{Intracellular survival assay}

INT407, RAW 264.7, Colo205 and peritoneal macrophage were seeded at a density of $2 \times 10^{5}$ cells per well in 24 -well plates. Stationary phase cultures of wild type and mutant Salmonella were added to the macrophages at a multiplicity of infection (MOI) of 10. For epithelial cell line infection, overnight grown culture was diluted at a ratio of $1: 33$ and incubated for $3 \mathrm{~h}$ to activate the SPI1 encoded genes required for invasion in non-phagocytic cells. The cells with bacteria containing media were centrifuged at $1000 \mathrm{rpm}$ for $10 \mathrm{~min}$ to facilitate entry of bacteria into cells. Plates were then incubated at $37^{\circ} \mathrm{C}$ for $25 \mathrm{~min}$ to allow bacterial uptake by cells. The extracellular bacteria were removed by washing thrice with PBS and incubating with $100 \mu \mathrm{g} / \mathrm{ml}$ gentamycin for $1 \mathrm{~h}$. Thereafter, the cells were incubated with $25 \mu \mathrm{g} / \mathrm{ml}$ gentamycin for the rest of the experiment. After 2 and $16 \mathrm{~h}$, the cells were lysed with $0.1 \%$ Triton-X 100 and the bacterial load was determined by plating serial dilutions of the lysates on LB agar plates with appropriate antibiotic. Colonies were enumerated after $24 \mathrm{~h}$.

\subsection{Salmonella burden in various organs}

BALB/c mice were bred and housed at the Central Animal facility, Indian Institute of Science. The mice used for the experiments were 6-8 weeks old. All procedures with animals were carried out in accordance with institution approved rules. Mice were starved for $12 \mathrm{~h}$ and then infected either orally $\left(10^{7} \mathrm{CFU}\right)$ or intraperitoneally $\left(10^{4} \mathrm{CFU}\right)$ with WT or $\triangle p m r G H-M D$ Salmonella. Mice were sacrificed after 4th and 7th day. Liver, mesenteric lymph node (MLN) and Peyer's patch (PP) were aseptically isolated, weighed and homogenized in PBS. The tissue debris were removed by centrifugation at $1500 \mathrm{rpm}$ for $5 \mathrm{~min}$. The bacterial load in the organs were determined by plating serial dilutions of the homogenate on LB-agar and SS agar (Salmonella-Shigella agar). SS agar was used only for Peyer's patch to select out the commensals present in small intestine. CFU were counted and represented as CFU/g wt. of organ.

\subsection{Immunization of mice through oral route}

Cohorts of five mice were orally inoculated under aseptic conditions with $1 \mathrm{ml}$ of PBS containing $10^{4} \mathrm{CFU}$ of $\triangle p m r G H-M D$. Placebo control mice were given only PBS. Three boosts at same doses were given at 1-week interval followed by final challenge with $10^{7} \mathrm{CFU} /$ mouse, WT Salmonella. Mice were sacrificed 5th day after challenge. Bacterial load in liver, spleen, MLN and PP were determined as described above. For assessing the safety of the vaccine in pregnant mice, cohorts of four pregnant mice were orally inoculated with $\triangle p m r G H-M D$ or WT S. typhimurium. Mice were scored for the delivery of the pups and their survival.

\subsection{Immunization of mice through intraperitoneal (i.p.) route}

Six- to eight-week-old female BALB/c mice were immunized via i.p. route with $1 \mathrm{ml}$ of PBS containing $10^{3} \mathrm{CFU}$ of $\triangle p m r G-H M-D$ strain per mouse in cohort of five mice. Booster doses were given on 7 th and 14th day. Placebo control mice were injected with PBS. On 21st day, challenge with either $10^{3} \mathrm{CFU} / \mathrm{mouse}$ (for organ load) or $10^{4}$ CFU/mouse (for scoring mortality) of WT Salmonella was done. Mice were sacrificed after 2 days. Bacterial load in liver, spleen, MLN and PP were determined as described above. Mortality of immunized and non-immunized mice was recorded.

\subsection{Immunization with single dose of vaccine and subsequent challenge}

Cohorts of five mice were immunized either orally or i.p. with $1 \mathrm{ml}$ of PBS containing $10^{3} \mathrm{CFU}$ of vaccine strain $(\Delta p m r G-H M-D)$ or PBS alone. The immunization was restricted to single dose only. After 42 days (oral route) and 
50 days (IP route), final challenge was performed with WT Salmonella at $10^{7}$ and $10^{3} \mathrm{CFU} /$ mouse, respectively. Mice were sacrificed after 4 days. Bacterial loads in liver, spleen, MLN and PP were determined as described above.

\subsection{CD4 and CD8 T cell population in spleen after immunization}

Cohorts of five mice were vaccinated orally and challenged as described before. Splenocytes from the spleen of vaccinated and control mice were isolated by homogenization. Homogenate were treated with RBC lysis buffer to remove RBC. Cells were counted and one million cells were used for FACS analysis (FACS scan analyser Becton, Dickinson and Company, USA). Cells were stained with antibody against CD4 and CD8 using hybridoma supernatants (kind gift from Prof. Dipanker Nandi, Department of Biochemistry, IISc) and Cy2 conjugated anti-rat secondary antibody. FACS analysis was done by using WinMDI software.

\subsection{Statistical analysis}

The data were subjected to statistical analysis by applying Student's $t$-test and Mann-Whitney test by using Sigma plot and Graph Pad prism 4 software and $p$-value $<0.05$ was considered as significant. All the experiments were repeated at least thrice to validate the results.
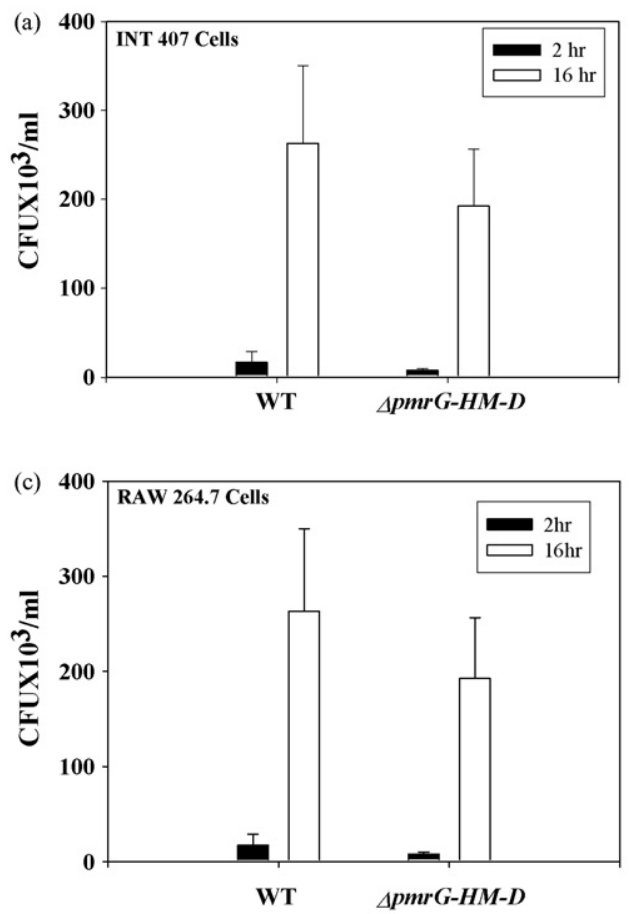

\section{Results}

\subsection{Construction and characterization of mutant}

pmrG-HM-D mutant was constructed by gene deletion through homologous recombination using $\lambda$-red recombinase system [14] (Fig. 1) and confirmed using colony PCR (data not shown). Long PCR using elongase showed product size of $8 \mathrm{~kb}$ for the pmrG-HM-D, whereas mutant showed product size of $1.1 \mathrm{~kb}$ corresponding to the chloramphenicol cassette. The growth characteristics of mutant with respect to WT remained the same in LB, M and F medium (data not shown).

$\Delta p m r G-H M-D$ strain was further characterized in terms of intracellular proliferation and survival in macrophage and epithelial cell line. In both the epithelial cell lines, INT407 and Colo205, $\Delta p m r G-H M-D$ was severely attenuated in terms of intracellular replication (Fig. 2a and b). While peritoneal macrophages showed a decreased survival of the mutant as compared to the WT from 2 to $16 \mathrm{~h}$, there was no visible difference in bacterial counts in the RAW264.7 macrophage cells with either strain (Fig. $2 \mathrm{c}$ and d).

\subsection{Organ load of pmrG-HM-D and wild type Salmonella}

To determine the organ load of wild type and $\Delta p m G$ $H M-D$ in mice, cohort of five mice were inoculated orally or
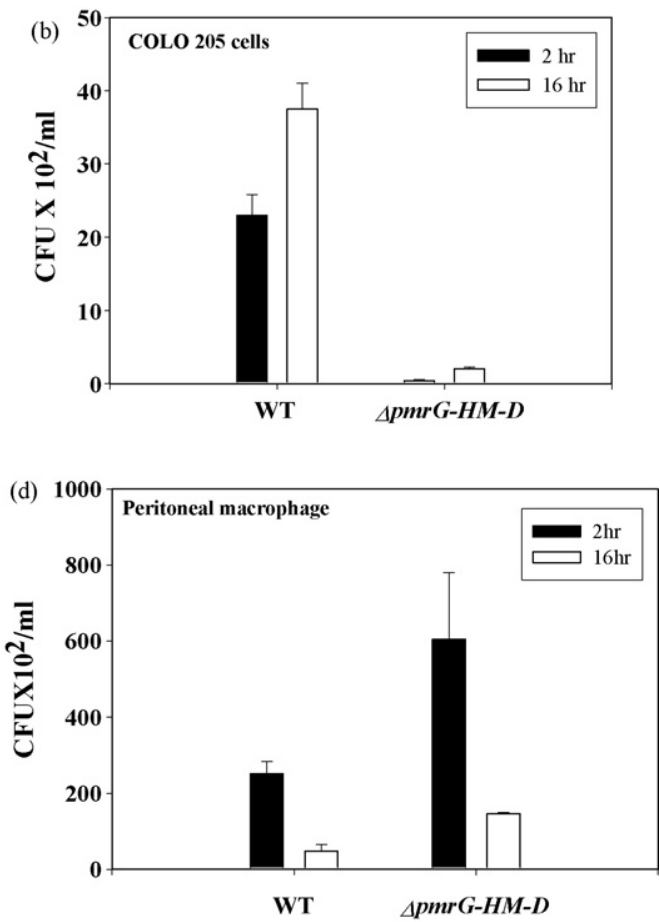

Fig. 2. Intracellular proliferation assays with various cell lines and primary peritoneal macrophage. Intracellular assay was performed with different cell lines. INT 407 (a), Colo205, (b) RAW 264.7 (c) and peritoneal macrophages (d) were infected with $\Delta p m r$-G-HM-D and wild type Salmonella. After 2 and 16 h, cells were lysed and serial dilutions of the lysates were plated on LB agar plates. All the experiments were done in triplicate. Data are depicted as mean $\mathrm{CFU} \pm$ S.D. 

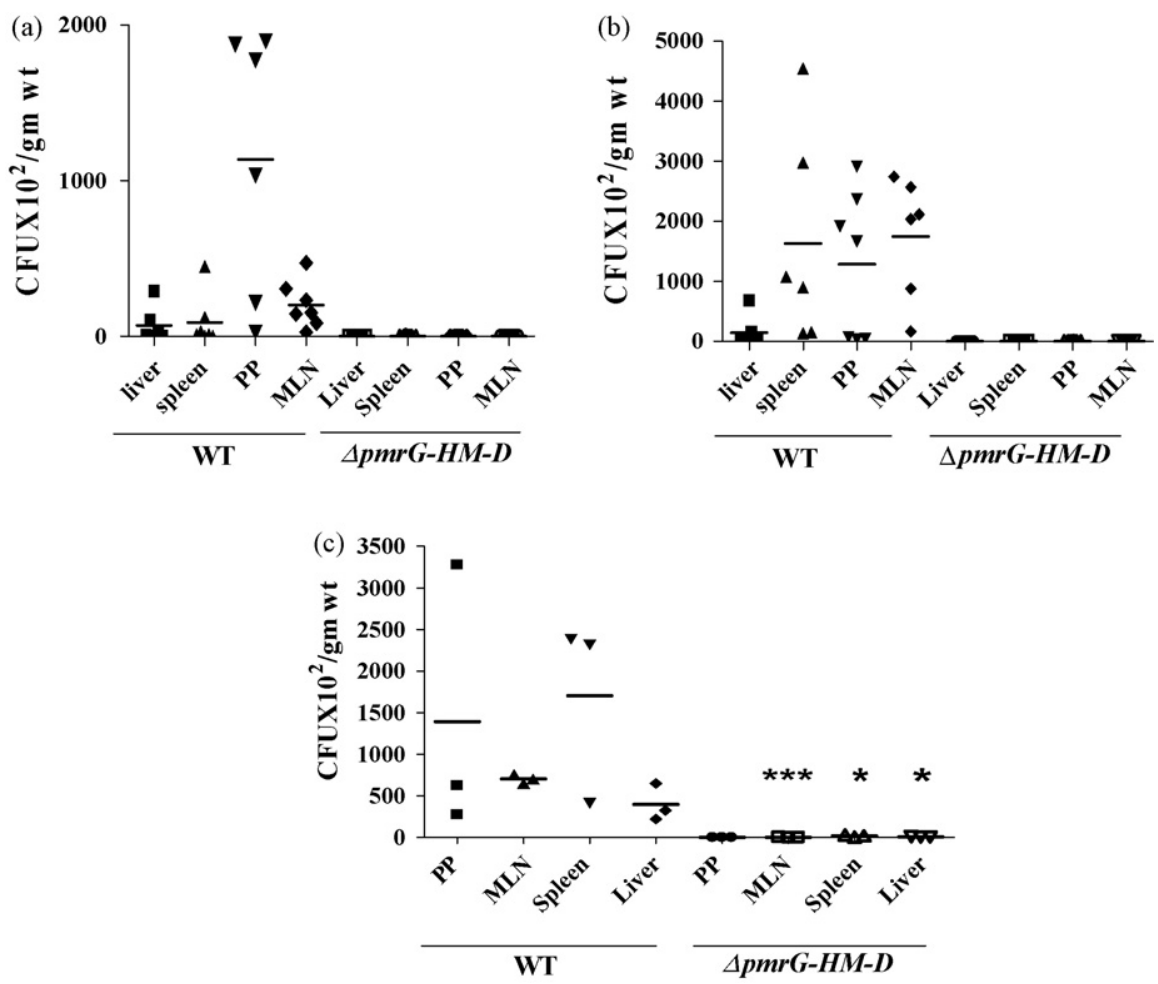

Fig. 3. Organ load of virulent Salmonella and the vaccine strain $\triangle p m r G-H M-D$. Two groups of mice were infected with $10^{7} \mathrm{CFU} / \mathrm{mouse}$ orally or $10^{3}$ CFU/mouse intraperitoneally and mice were sacrificed on day 4 (a) and day 7 (b) after oral infection and on day 3 (c) after i.p. infection. Liver, spleen, PP and MLN were aseptically dissected. Tissues were weighed and homogenized. Serial dilution of homogenized samples were plated on LB agar plates and on SS plates (PP only). The plates were incubated for $24 \mathrm{~h}$ and the colonies were counted. The Mann-Whitney U test was used to compare the CFU between vaccinated and non-vaccinated mice. For all statistical analyses, $p<0.05$.

intraperitoneally with $10^{7}$ and $10^{4} \mathrm{CFU}$, respectively. After 4th and 7th day, Salmonella burden was evaluated from various organs. As shown in Fig. 3a and b, WT Salmonella was able to survive and proliferate in PP, spleen, MLN and liver after 4th and 7th day of infection. In contrast, $\triangle p m r G-H M$ $D$ was severely attenuated. Almost $100-1000$ fold reduction in the CFU from various organs was observed in mice inoculated with $\triangle p m r G-H M-D$ strain as compared to WT. This suggested that the mutant is efficiently cleared from the PP and the secondary lymphoid organs. As depicted in Fig. 3c, the attenuation of the vaccine strain was also observed during intraperitoneal route of infection after third day of infection. PmrA and PmrHIFJKLM mutant were found to be attenuated only when given through oral route and not through intraperitoneal route [13]. However, our multiple mutant vaccine strain could neither survive the harsh environment of the small intestine nor the peritoneal cavity. We further investigated whether this clearance could generate protective immunity when challenged with WT Salmonella.

\subsection{Multiple immunizations with pmrG-HM-D mutant was able to rescue the mice}

$\mathrm{BALB} / \mathrm{c}$ mice were primed orally with the $\triangle p m r G-H M$ $D$ followed by two booster doses on 7 th and 14th day, all at doses of $10^{4} \mathrm{CFU} / \mathrm{mouse}$. After 7 th day of the final boost,

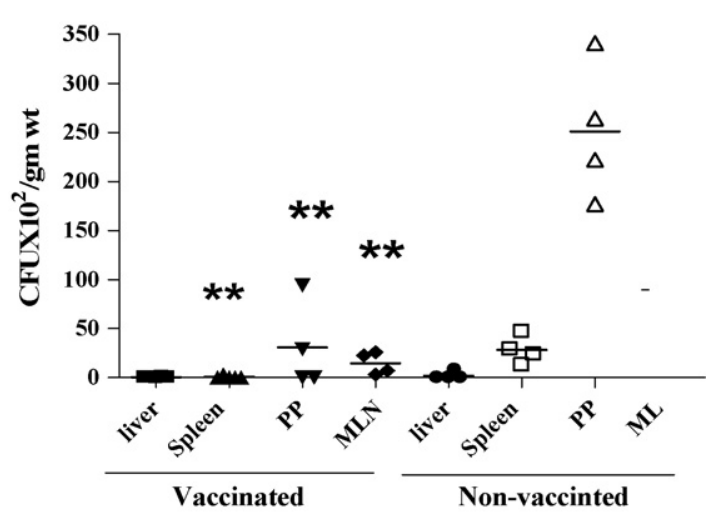

Fig. 4. Oral immunization with $\triangle p m r G-H M-D$ reduced the burden of WT Salmonella in liver, spleen MLN and PP. Six- to eight-week-old female $\mathrm{BALB} / \mathrm{c}(n=10)$ were orally immunized with $10^{4} \mathrm{CFU}$ of $\triangle p m r G-H M-D$ or PBS, boosted twice on days 7 and 14 and orally challenged with $10^{7}$ CFU of WT $S$. typhimurium. On day 4 post-challenge, mice were sacrificed and immediately necropsied using standard techniques. The number of Salmonella present in the mesenteric lymph nodes (MLN), spleen and PP was enumerated using direct cultures of serially diluted homogenized samples. The Mann-Whitney $U$-test was used to compare CFU in various organs between vaccinated and non-vaccinated mice. For all statistical analyses, $p<0.05$. 

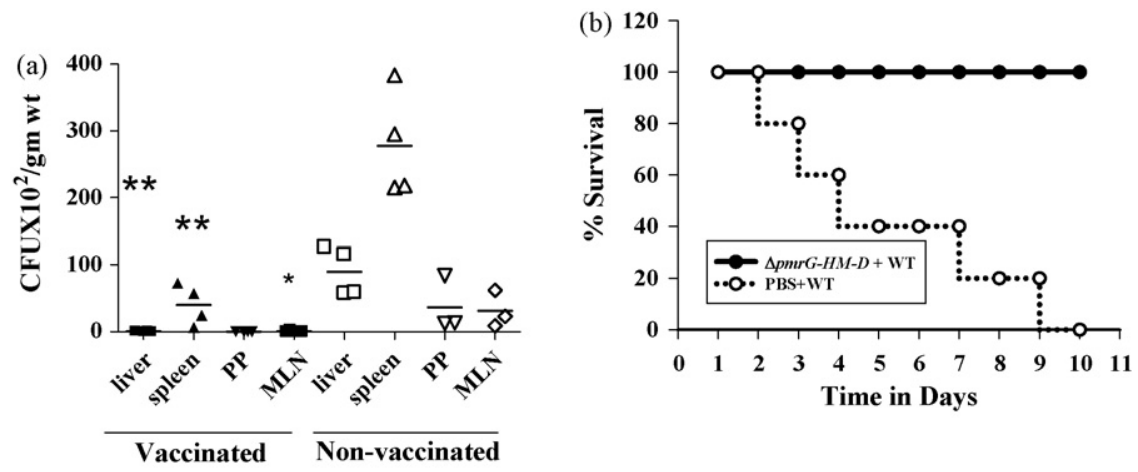

Fig. 5. Intraperitoneal immunization with $\triangle p m r G-H M-D$ conferred protection against WT colonization of liver, spleen MLN and PP in mice. Six- to eightweek-old female BALB/c $(n=10)$ were immunized through i.p route with $10^{3} \mathrm{CFU}$ of $\triangle p m r G-H M-D$ or PBS, boosted twice with the same dose on days 7 and 14 and challenged with either (a) $10^{3} \mathrm{CFU}$ of WT S. typhimurium for assessing organ load or (b) $10^{4} \mathrm{CFU}$ of WT S. typhimurium for assessing mortality. On second day post-challenge, mice were sacrificed and immediately necropsied using standard techniques. The number of salmonellae present in tissues mesenteric lymph nodes (MLN), spleen and PP was enumerated using direct cultures of serially diluted homogenized samples. The Mann-Whitney $U$-test was used to compare $\mathrm{CFU}$ in various organs between vaccinated and non-vaccinated mice. For statistical analyses, ${ }^{*} p<0.05,{ }^{* *} p<0.005$.

mice were challenged with $10^{7} \mathrm{CFU}$ of wild type Salmonella. As shown in Fig. 4, in the immunized mice, there was 100fold reduction in the bacterial load in spleen, MLN and PP as compared to the organs of placebo control mice.

Intra-peritoneal route of Salmonella infection is very lethal, requiring as low as $10^{3} \mathrm{CFU}$ for mortality of the mice. BALB/c mice were primed with $10^{3} \mathrm{CFU} \Delta p m r G$ -
$H M-D$, followed by two boosters on 7th and 14th day and finally infected with $10^{7} \mathrm{CFU}$ of wild type Salmonella. There was marked decrease in organ CFU of liver, spleen and MLN in vaccinated mice as compared to control unprimed mice (Fig. 5a). Priming with $\triangle p m r G-H M-D$ was also able to decrease the splenomegaly usually caused by virulent Salmonella infection (data not shown). As depicted in Fig. 5b,
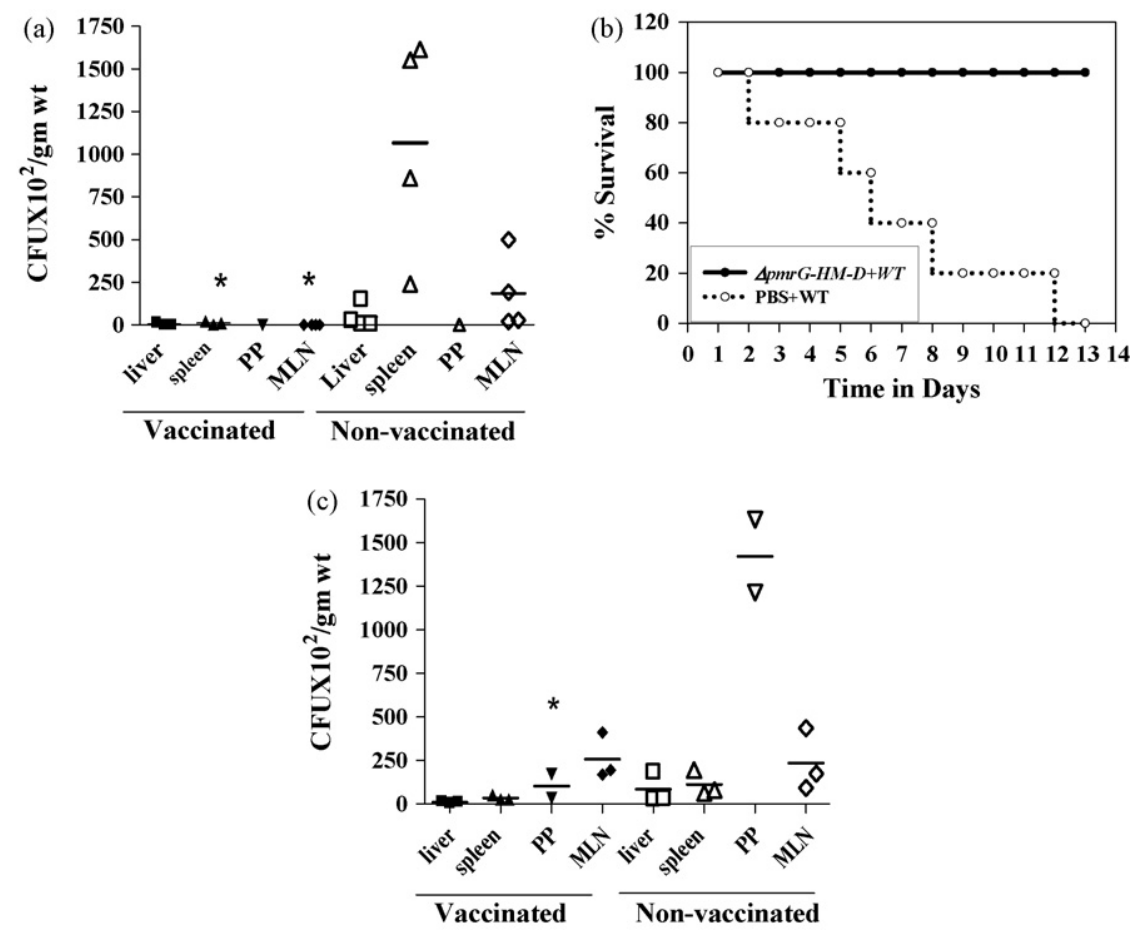

Fig. 6. Single dose of vaccination was able to rescue completely the challenged mice after 50 days of immunization. Each group of five mice were vaccinated via i.p. route and given final lethal challenge of $10^{4} \mathrm{CFU} /$ mouse for mortality and $10^{3}$ per mouse for organ load. (a) Organ CFU in mice when challenged with WT CFU of $10^{3}$ per mouse. (b) Mouse viability after lethal challenge of $10^{4} \mathrm{CFU} /$ mouse showing complete rescue from infection. (c) Two groups of five mice each were vaccinated orally with either vaccine strain $(\Delta p m r G-H M-D)$ or PBS and subsequently challenged with WT Salmonella after 42 days. On day 4 after challenge, mice were sacrificed and immediately necropsied using standard techniques. The number of salmonellae present in tissues mesenteric lymph nodes (MLN), spleen and PP was enumerated using direct cultures of serially diluted homogenized samples. The Mann-Whitney $U$ test was used to compare CFU in various organs between vaccinated and non-vaccinated mice. For statistical analyses, ${ }^{*} p<0.05,{ }^{* *} p<0.005$. 
Table 2

Safety of serovar Typhimurium vaccine strains after oral administration to pregnant mice

\begin{tabular}{llll}
\hline No. of pregnant mice & Relevant genotype & No. of abortions & $\begin{array}{c}\text { No. of mice (healthy/dead) } \\
\text { (no. of death/total no.) }\end{array}$ \\
\hline 4 & Wild type & 1 & $3 / 1$ \\
4 & $\Delta p m r G-H M-D$ & 0 & $4 / 0$ \\
\hline
\end{tabular}

the vaccinated mice showed $100 \%$ survival upon lethal dose challenge with wild type Salmonella. On the other hand, all the non-vaccinated mice succumbed to death by 13 th day of infection.

The excellent protection conferred by $\Delta p m r G-H M-D$ suggests that the evasion strategy, Salmonella uses to escape the defense mechanism of the host is abrogated by this vaccine strain.

\subsection{Single dose of pmrG-HM-D mutant protected mice from subsequent infection}

The number of doses required for vaccination is an important factor for determining the efficiency of the vaccine. Here, we show that vaccination of mice with single dose of $\Delta$ pmrG-HM-D, was able to decrease the organ burden when challenged with wild type Salmonella, after 50 days (IP vaccination and IP challenge) (Fig. 6a) and 42 days (oral vaccination and oral challenge) (Fig. 6c). Moreover, single dose of vaccination and challenge after 50 days with wild type Salmonella showed $100 \%$ protection (Fig. 6b).

\subsection{Effect of pmrG-HM-D mutation on the capacity to prime $C D 8+$ and $C D 4+T$ cell responses}

We further evaluated whether the $p m r G-H M-D$ mutation affects the modulation of CD4+ and CD8+ T cell response by Salmonella. To test this, mice were vaccinated with the $\Delta$ pmrG-HM-D and challenged with WT Salmonella. Spleen cells were analyzed for the CD8+ and CD4+ T cell counts. The mice injected with $\triangle p m r G-H M-D$ strain showed an increase in the CD8+ $\mathrm{T}$ cell population unlike the WT strain (Fig. 7a). Mice vaccinated with $\triangle p m r G-H M-D$ and then challenged with WT exhibited a marked increase from $0.55 \%$ (WT) to $11.44 \%$ (vaccinated and challenged) of CD8+ T cells but not CD4+ T cells (Fig. 7b). Taken together these data suggest that probably, pmr systems play an important role in immune evasion mechanism by inhibiting $\mathrm{CD} 8+\mathrm{T}$ cell response.

\subsection{Safety of the vaccine strain in pregnant mice model}

To assess the possibility of pregnancy failure after challenge with the vaccine strain and WT, groups of four pregnant mice were inoculated orally at 12-15 days of pregnancy. As shown in Table 2, WT virulent $S$. typhimurium strain caused abortion in one mouse and killed one mouse. Among the pups delivered by the remaining two mice, $50 \%$ pups died within
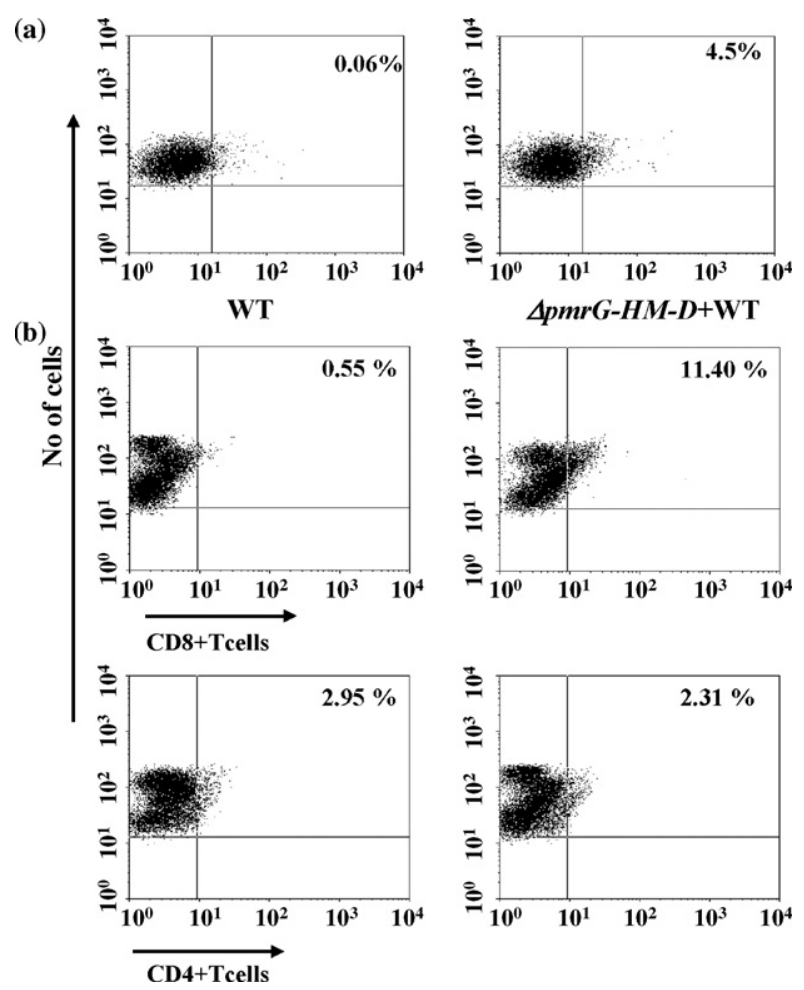

Fig. 7. CD8+ T cell population in vaccinated and non-vaccinated mice. (a) $\mathrm{BALB} / \mathrm{c}$ mice were given intraperitoneal injection of $10^{3} \mathrm{CFU}$ of either WT or vaccine strain $(\triangle p m r G-H M-D)$. After third day of injection, mice were sacrificed and spleen cells were processed for presence of CD8+ T cells by FACS analysis. (b) BALB/c mice were vaccinated intraperitoneally with $10^{3} \mathrm{CFU}$ of vaccine strain on days 7, 14 and 21 . Placebo control mice were injected with PBS alone. Mice were challenged with $10^{3} \mathrm{CFU}$ of WT and on third day, spleen were processed for presence of CD8+ and CD4+ T cells by FACS analysis. CD4+ and CD8+ T cells were represented as \% positive cells.

a period of $24 \mathrm{~h}$ after birth. On the other hand, the pregnant mice inoculated with the vaccine strain $\triangle p m r G-H M-D$ were all healthy and delivered healthy pups. Taken together, these data suggest that the vaccine strain $\triangle p m r G-H M-D$ is safe during pregnancy.

\section{Discussion}

In the present scenario, Salmonella vaccine is known as a fruit not to be eaten. Various research groups are trying to develop a better vaccine which can be well tolerated in children and have better protective immunity. The extra-intestinal infections of Salmonella, are very rare but life threatening [14]. With increase in the antibiotic resistant strains, vaccine remains the only choice to curb this disease. Diseases 
of the Most Impoverished (DOMI) Program, was launched in 1999 to accelerate the development and use of current and future new-generation vaccines against typhoid fever [15]. This implies the urgent need for a potent vaccine against typhoid fever.

Salmonella is an intracellular pathogen having various strategies to evade the defense mechanism of the host. Murine model is an excellent system available to study the pathogenesis of typhoid fever caused by S. typhimurium. Researchers all over the world are trying to create an ideal vaccine strain which could give long lasting protective immunity. The genes of choice to be deleted were either metabolic or pathogenicity island genes like aroA, purD or SPI2. These vaccine strains were attenuated but were not able to protect the host. We hypothesized that, deleting a set of genes which are thought to play an important role in counteracting the environmental danger signals like AMPs may facilitate the killing of Salmonella thereby giving protective immunity. The intricate relation with PmrA-B (two component regulatory system), PmrD and PmrG enable one system to respond to multiple signals, which is important for the bacteria to adapt to the harsh environment of the host. These genes are responsible for the LPS modifications, resistance to AMPs and Fe(III). Deletion of these genes may lead to the susceptibility of the Salmonella strains to various AMPs as well as other host environment which may be rich in iron. Hence, in this study we have created a strain which is deficient in the PmrD, Pmr $\mathrm{H}-\mathrm{M}$ and PmrG genes. This multiple mutant strain, $\Delta p m r G$ $H M-D$, provided excellent protection in the animal model of typhoid fever. Loss of expression of the pmrHIFJKLM operon eliminates Ara4A addition to lipid A and polymyxin B resistance [16]. PmrD is hypothesized to interconnect PmrAB and Pho P-Q system [12]. It was recently demonstrated that dephosphorylation of one of the phosphates in the core region of the LPS by the PmrA-activated PmrG protein confers Fe(III) resistance [17]. Single deletion of pmrA and pmrHIFJKLM operon, resulted in a attenuated Salmonella for the oral route but not intraperitoneal route [16]. All these studies suggest that probably these genes are necessary for the intestinal invasion and survival. The multiple mutant vaccine strain, $\Delta p m r G-H M-D$ was able to disarm the virulence strategies which may be hindering the protective immunity in case of typhoid fever. This multiple vaccine strain may have defective LPS structure with more susceptibility to AMPs and may have dysregulation of the phoP regulon. Defect in the phoP regulon leads to attenuation of Salmonella in the intraperitoneal route of infection. This vaccine strain was not only attenuated when given through oral route, but also through intraperitoneal route.

$\Delta p m r G-H M-D$ strain was not only efficient in conferring excellent protection against Salmonella challenge, but also required less dose $\left(10^{4} \mathrm{CFU}\right)$ as compared to other vaccine strains, which were given at doses ranging from $10^{5}$ to $10^{8}$ CFU [18-20]. $\Delta p m r-G-H M-D$ strain was markedly attenuated in epithelial cells. The epithelial cells are rich in antimicrobial peptides (AMP), which may probably explain the drastic decrease in intracellular proliferation rate of this mutant in epithelial cells as observed in our study. However, $\triangle p m r G-H M-D$ strain was comparable in the in vitro growth characteristics with respect to wild type Salmonella. This suggests that the LPS modifications and loss of regulation of other virulence genes, may be responsible for the attenuation of this strain in the mouse model. Vaccination of the mice with $\triangle p m r G-H M-D$ strain not only decreased the organ burden of wild type Salmonella but also conferred $100 \%$ protection against the challenge. Being an intracellular pathogen, development of strong cell mediated immune response will a primary requisite to clear the infection. The vaccinated mice exhibited appreciable increase in CD8+ T cells suggesting its role in protection. Further in vitro studies are in progress to look more closely into the functionality of these CD8+ T cells, in terms of CTL response and IFNy production. Also, studies are underway to elucidate the memory population of CD8+ T cells, which can contribute to the long-term protective immunity against Salmonella. One more important observation was the induction of abortion in pregnant mice by the WT Salmonella and not by vaccine strain, $\Delta p m r G-H M-D$. Moreover, the pups which were delivered from the WT infected mice also died a day after delivery. The vaccine strain neither induced abortion nor caused death of the pups. These finding strongly suggests that the vaccine strain $\triangle p m r G-H M-D$ appears to be safe even in pregnant individuals.

An ideal live vaccine strain combines efficient immunogenicity with safety. In this study, we have shown that a $\Delta p m r G-H M-D$ mutant of $S$. enterica serovar Typhimurium fulfills both of these criteria. The mutant becomes attenuated and elicits protective immunity against a subsequent challenge with the wild type strain, requires a single low dose and is very safe even in the pregnant animal model.

\section{Acknowledgments}

We thank Prof. Micheal Hensel (FAU, Erlangen, Germany) for providing Salmonella wild type strain. We thank Prof. R. Nayak, Prof. M.S. Shaila, Dr. Nandakumar, Ananthalaxmi, Dr. Sandeepa and Dr. Arvindhan for their critical comments. We thank Dr. Omana Joy for the FACS analysis. This work was supported by grant, Provision (2A), Tenth Plan (191/MCB) from the Director of Indian Institute of Science. The facilities under ICMR center for Medical Microbiology and DBT program support on Basic Biology of Microbial Pathogens are highly acknowledged.

\section{References}

[1] Synder JW, Check W. Bioterrorism threats to our future. A report from American Society of Microbiology; 2000.

[2] Crump JA, Luby SP, Mintz ED. The global burden of typhoid fever. Bull World Health Organ 2004;82:346-53. 
[3] Bhan MK, Bahl R, Bhatnagar S. Typhoid and paratyphoid fever. Lancet 2005;366:749-62.

[4] Kirkpatrick BD, McKenzie R, O'Neill JP, et al. Evaluation of Salmonella enterica serovar Typhi (Ty2 aroC-ssaV-) M01ZH09, with a defined mutation in the Salmonella pathogenicity island 2, as a live, oral typhoid vaccine in human volunteers. Vaccine 2006;24: 116-23.

[5] Khan SA, Stratford R, Wu T, et al. Salmonella typhi and $S$ typhimurium derivatives harbouring deletions in aromatic biosynthesis and Salmonella Pathogenicity Island-2 (SPI-2) genes as vaccines and vectors. Vaccine 2003;21:538-48.

[6] Tacket CO, Sztein MB, Losonsky GA, Wasserman SS, Nataro JP, Edelman R, et al. Safety of live oral Salmonella typhi vaccine strains with deletions in htrA and aroC aroD and immune response in humans. Infect Immun 1997;65:452-6.

[7] Ivanoff B, Levine MM, Lambert PH. Vaccination against typhoid fever: present status. Bull WHO 1994;72:957.26.

[8] Miller SI, Loomis WP, Alpuche-Aranda C, Behlau I, Hohmann E. The PhoP virulence regulon and live oral Salmonella vaccines. Vaccine 1993;11(2):122-5.

[9] Yrlid U, Svensson M, Hakansson A, Chambers BJ, Ljunggren HG, Wick MJ. In vivo activation of dendritic cells and $\mathrm{T}$ cells during Salmonella enterica serovar Typhimurium infection. Infect Immun 2001;69:5726-35.

[10] Sebastiani G, Blais V, Sancho V, Vogel SN, Stevenson MM, Gros $\mathrm{P}$, et al. Host immune response to Salmonella enterica serovar Typhimurium infection in mice derived from wild strains. Infect Immun 2002;70:1997-2009.

[11] Trezena AG, Souza CM, Borrego A, Massa S, Siqueira M, De Franco $\mathrm{M}$, et al. Co-localization of quantitative trait loci regulating resistance to Salmonella typhimurium infection and specific antibody production phenotypes. Microbes Infect 2002;4:1409-15.
[12] Eguchi Y, Utsumi R. A novel mechanism for connecting bacterial twocomponent signal-transduction systems. Trends Biochem Sci 2005;30: $70-2$.

[13] Datsenko KA, Wanner BL. One-step inactivation of chromosomal genes in Escherichia coli K-12 using PCR products. Proc Natl Acad Sci USA 2000;97(June (12)):6640-5.

[14] Huang DB, DuPont HL. Problem pathogens: extra-intestinal complications of Salmonella enterica serotype Typhi infection. Lancet Infect Dis 2005;5:341-8.

[15] DeRoeck D, Clemens JD, Nyamete A, Mahoney RT. Policymakers' views regarding the introduction of new-generation vaccines against typhoid fever, shigellosis and cholera in Asia. Vaccine 2005;23: 2762-74.

[16] Gunn JS, Ryan SS, Van Velkinburgh JC, Ernst RK, Miller SI. Genetic and functional analysis of a PmrA-PmrB-regulated locus necessary for lipopolysaccharide modification, antimicrobial peptide resistance, and oral virulence of Salmonella enterica serovar Typhimurium. Infect Immun 2000;68:6139-46.

[17] Nishino K, Hsu FF, Turk J, Cromie MJ, Wosten MM, Groisman EA. Identification of the lipopolysaccharide modifications controlled by the Salmonella PmrA/PmrB system mediating resistance to Fe(III) and Al(III). Mol Microbiol 2006;61:645-54.

[18] Nagy G, Dobrindt U, Hacker J, Emody L. Oral immunization with an rfaH mutant elicits protection against salmonellosis in mice. Infect Immun 2004;72:4297-301.

[19] Tang IK, Ji DD, Chou CF, Lin HC, Liao CL, Sytwu HK, et al. Characterization of a highly attenuated Salmonella enterica serovar Typhimurium mutant strain. J Microbiol Immunol Infect 2002;35:229-35.

[20] Valentine PJ, Devore BP, Heffron F. Identification of three highly attenuated Salmonella typhimurium mutants that are more immunogenic and protective in mice than a prototypical aroA mutant. Infect Immun 1998;66:3378-83. 